would be substantial. Relatives would gain greater understanding of schizophrenia and thus be better able to help patients acquire self-knowledge. Greater independence and fewer relapses should result. Professional carers would find their work more rewarding: 'community care' would become less of a slogan and more of a true interaction of benefit to those who badly need help.

The principles of specific (as opposed to general) health education cannot be laid down as systematically or precisely as they can for conditions such as diabetes or even dementia. But we now have sufficient knowledge to ensure that caring relatives who wish for, and can cope with, such help can become reasonably well-informed. To make this information readily available should be one of the major tasks of psychiatrists and of the College.

REFERENCE

'RoLLIN, H. (ed) (1980) Coping with Schizophrenia: The National Schizophrenia Fellowship. London: Burnett Books and Andre Deutsch.

\title{
Limiting the Range of Drugs Prescribable under the NHS
}

After the meeting of Council on 15 February 1985, the President sent the following comments on the Government's proposals to Mr Norman Fowler, Secretary of State for Social Services.

(1) The College is in favour of rationalizing the prescribing of drugs and ensuring the best use of finite resources, but believes that there are better ways of achieving the desired results. We have serious reservations about the effect of the proposals on the care of our patients and we wish to object to the unrealistically short period of consultation offered.

(2) The College is opposed to a statutory 'restricted list' As well as being unacceptable in principle, it is anticipated that such a list would require cumbersome and continuous revision and a complicated appeals machinery. Paradoxical situations can be envisaged in which special arrangements would have to be made for private prescriptions to be dispensed for NHS hospital in-patients.

(3) The College is opposed to proposals that limit the drugs available in NHS practice while allowing a wider range in private practice. Doctors will be faced with a difficult ethical conflict between prescribing the best treatment available for patients and prescribing the treatment the patient can afford.

(4) The proposals put forward are unacceptably rigid in view of the range of widely differing clinical conditions that are treated with one compound and of the complex and varied nature of compounds that may be needed to treat a single clinical condition. For instance, in the case of benzodiazepines, what applies to the treatment of a neurosis does not necessarily apply to treatment of epilepsy or spastic paraplegia, nor is appropriate to their use in intubation procedures. Benzodiazepines vary widely in their profile of action, their formulation and aspects of pharmacokinetics. Moreover, the pharmacokinetics of drugs varies in sometimes unpredictable ways, e.g. in the elderly. The suggestion that the proposals affect only minor and self-limiting complaints is unfortunate. Constipation in elderly patients can be a major clinical problem. A wide range of laxatives is also required as part of the treatment of more serious conditions (for example, constipation caused by opiates in patients with terminal cancer).

(5) The present proposals carry the inherent and unacceptable risk that more dangerous drugs might be prescribed in place of the restricted drugs (e.g. barbiturates or neuroleptics instead of benzodiazepines or opiates instead of aspirin).

(6) The College is concerned that this proposal will not only cause extra expenditure for sick and needy patients, but that some patient groups, whose prescriptions are free at present, would have to pay the full cost of the drug in future.

\section{'Speculations in Science and Technology'}

Speculations in Science and Technology publishes speculative papers in the physical, mathematical, biological and engineering sciences, together with contributions in interdisciplinary areas. It is published five times a year.
Submissions are now invited and should be sent to the Editor, Dr Alan L. Mackay, Department of Crystallography, Birkbeck College, Malet Street, London WC1E 7HX. 